\title{
Artificial Muscles of Dielectric Elastomers Attached to Artificial Tendons of Functionalized Carbon Fibers
}

\author{
Zhihang $\mathrm{Ye}^{1}$, Md. Shahnewaz Sabit Faisal ${ }^{2}$ \\ Faculty: Zheng Chen ${ }^{1}$, Ramazan Asmatulu ${ }^{2}$ \\ ${ }^{1}$ Department of Electrical Engineering and Computer Science, College of Engineering \\ ${ }^{2}$ Department of Mechanical Engineering, College of Engineering
}

\begin{abstract}
Dielectric elastomers (DEs) are soft actuation materials with promising applications in robotics and biomedical devices. In this paper, a bio-inspired artificial muscle actuator with artificial tendons is developed for robotic arm applications. The actuator uses a dielectric elastomer as artificial muscle and functionalized carbon fibers as artificial tendons. A VHB 4910 tape is used as the dielectric elastomer and PDMS is used as the bonding material to mechanically connect the carbon fibers to the elastomer. Carbon fibers are highly popular for their high electrical conductivities, mechanical strengths, and biocompatibilities. After the acid treatments for the functionalization of carbon fibers $(500 \mathrm{~nm}-10 \mu \mathrm{m})$, one end of carbon fibers is spread into the PDMS material, which provides enough bonding strength with other dielectric elastomers, while the other end is connected to a DC power supply. Experimental results show that the conductivity of carbon fibers is capable of transfer input. The bonding strength of carbon fibers and PDMS material is also large enough to pull external load.
\end{abstract}

\section{Introduction}

Many soldiers and civilians lose their hands, arms and legs in the battle fields, accidents, and natural disasters. Many senior people also lose the capabilities of their arms and legs due to some diseases and aging process. Research has been conducted to develop robotic assistive devices, such as prosthetic arms to help those disabled people. However, most of the existing prosthetic arms are driven by electric motors, which are heavy and rigid. Soft, lightweight, and energy efficient artificial muscles are highly needed for prosthetic arm applications.

Dielectric elastomer is an important category of artificial muscles, which can generate large deformation with high force output. A typical DE actuator usually consists of a sandwiched structure [1]. Two layers of conductive and stretchable materials are coating on both sides of a DE material layer. When a voltage is applied on the actuator, the PDMS material will compress in thickness and stretch in area [2, 3]. The main problem of this structure is the poor electrical and mechanical interfaces, which limits its application in prosthetic arm.

In this paper, artificial tendons are introduced in the artificial muscle structure. In the proposed structure, a DE material is used as artificial muscle to generate mechanical output under electrical voltage input. Carbon fibers are used as artificial tendons to transfer electrical input and mechanical output simultaneously. Carbon fibers are highly conductive material, which can be used to connect the electrical input to the artificial muscle. In other words, carbon fibers function as an electrical and mechanical interface.

\section{Design of Artificial Muscle with Artificial Tendon}

The goal of this research is to build dielectric elastomer actuator connected with artificial tendon. A VHB tape (VHB 4910, 3M) is used as the dielectric elastomer and carbon fibers are used as artificial tendon. Figure 1 shows the cross-sectional view of the actuator design. The actuator can be divided into working area and contact area. In the working area, carbon particles are coated on both sides of the tape as compliant electrodes. In the contact area, two clusters of carbon fibers are bonded by PDMS (Ecoflex 0030, Smooth-on Inc.) on different sides, and are connected to the electrodes of working area.

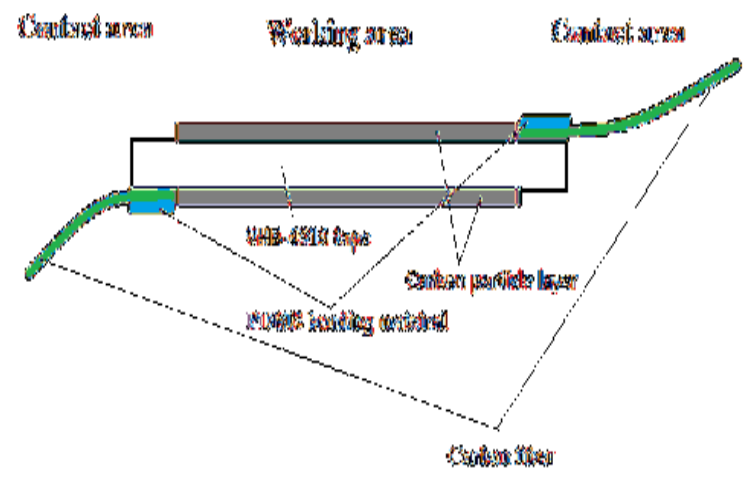

Fig. 1. Design of Artificial Muscle. 


\section{Fabrication and Experimental Test}

Before realizing the artificial muscle, two intermediate devices were made to characterize the actuation capability of the DE material and verify the electrical conductivity and mechanical strength of the carbon fibers.

First, a diaphragm actuator (shown in Fig. 2) was built to test the carbon fibers' conductivity and characterize the DE material's working performance. The VHB tape was pre-stretched by about $30 \%$. Both sides of the tape were pasted with carbon black. Two clusters of carbon fibers were attached on each side of the actuator by sticky tape. A calibration weight was put on its center as load. When the input was turned on, the load moved down. The load returned back to its initial state when the input was off.

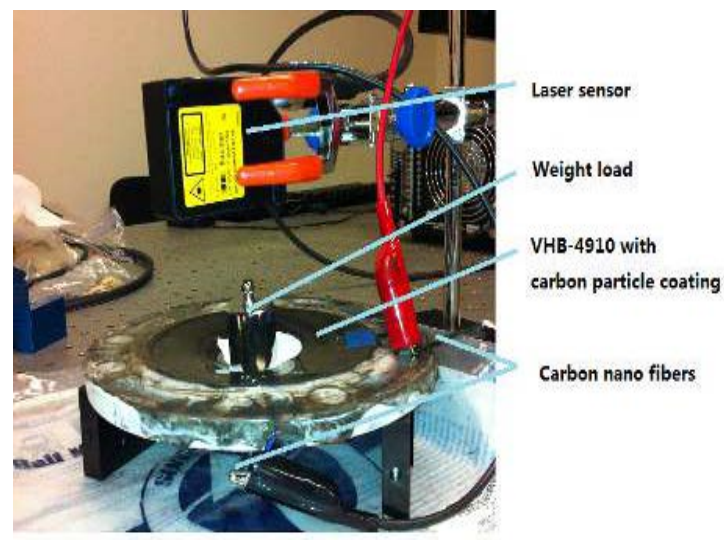

Fig. 2. Diaphragm Actuator and Experimental Setup.

A laser sensor (OADM 20I6441/S14F, Baumer) was used to measure the displacement of the load. A dSPACE real-time system was used to generate voltage signal. A high voltage amplifier (PS350, Stanford Research System INC) was used to amplify the voltage signal. Actuation voltage and current were measured by the dSPACE. Fig. 3 shows the step response of the actuator under different weight loads, while the step voltage remains $3 \mathrm{KV}$. The step response shows that the actuator has about $0.5 \mathrm{sec}$ response delay and 1 sec settling time. The heavier the load, the longer the settling time.

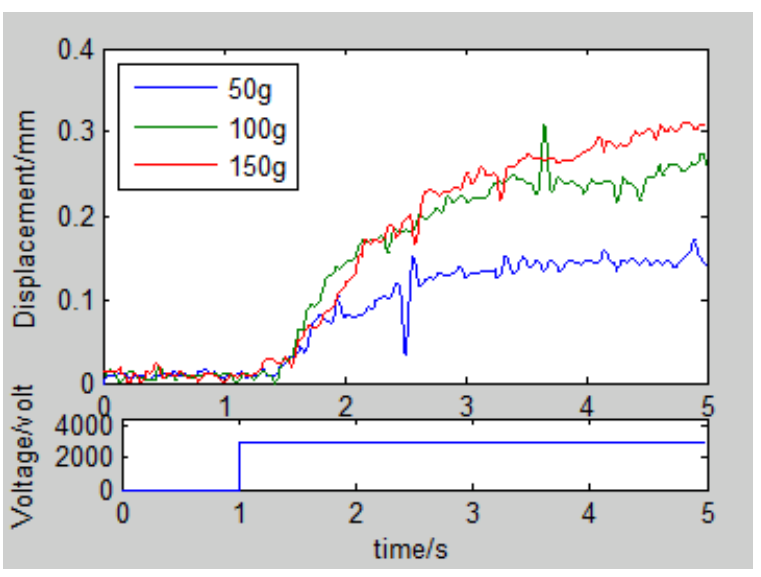

Fig. 3. Step Responses under 3 KV with Different Loads.

Second, a strip PDMS/Carbon Fiber specimen (Shown in Fig. 4) was fabricated to verify the bonding strength between artificial tendon and PDMS material. Carbon fibers were placed into am acrylic mold and PDMS was poured into the mold. A vacuum was used to get rid of the bubbles in the rubber liquid. Then the PDMS was cured overnight under room temperature. Tension tests are simply implemented by human hands. The specimen can be dragged by the full length of human arm. After removal of external force, it comes back to its original state. Which means the bonding strength is strong enough for prosthetic arms during daily use.

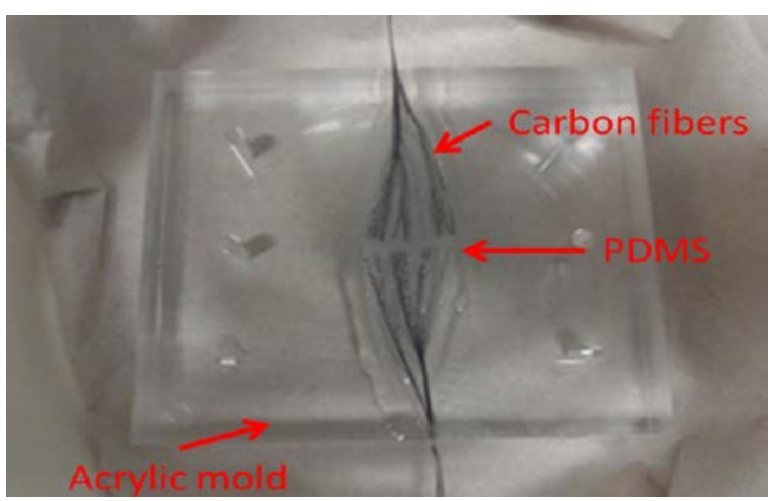

Fig. 4. Strip Specimen in the Mold.

\section{Conclusion}

In this paper, a bio-inspired artificial muscle actuator with artificial tendon was developed. Two devices have been fabricated to verify the suitability of using carbon fibers as artificial tendons. First, a diaphragm dielectric actuator has been fabricated and characterized. It shows that carbon fibers are conductive enough and can acts as the electrical input interface. It also characterizes the actuation 
performance of the dielectric elastomer. Second, specimen of PDMS bonded with carbon fibers has been fabricated and tested. It shows that the mechanical bonding between PDMS and carbon fiber is strong enough for robotic arm applications.

\section{Acknowledgement}

This research was supported in part by the Kansas Engineering Expansion Grant.

\section{References}

[1] R. Pelrine, R. Kornbluh, J. Joseph, R. Heydt, Q. Pei, and S. Chiba, "High-field deformation of elastomeric dielectrics for actuators,” Materials Science and Engineering C 11, pp. 89-100, 2000.

[2] Z. Suo, "Theory of dielectric elastomers," Acta Mechanica Solida Sinica 23, pp. 549-578, 2010.

[3] L. Liu, Y. Liu, and J. Leng, "Theory progress and applications of dielectric elastomers," International Journal of Smart and Nano Materials 4(3), pp. 199-209, 2013. 